\section{I2 Einteilung von Medizinprodukten}

S. Klein, Lübeck; M. Kraft, Berlin

Die direkte Anwendung und Wirkung medizintechnischer Systeme am lebenden Objekt kann generell mittels verschiedener Charakteristika beschrieben werden, anhand derer auch eine Einteilung von Medizinprodukten möglich ist [1]. Zu den Einteilungskriterien gehören:

- die Invasivität der Informationserfassung bzw. des Eingriffs:

- invasiv (dringt durch Körperoberfläche oder über eine Körperöffnung ganz oder teilweise in den Körper ein), z. B. Herzschrittmacherimplantation, Messung des zentralvenösen Blutdrucks, tiefe Hirnstimulation,

- nichtinvasiv, z. B. Ultraschallbildgebung, OberflächenEKG,

- nach dem Ort der Anwendung bzw. der Art des Verfahrens:

- in vivo (im/am lebenden Objekt), z. B. Operationsmikroskopie,

- in situ (in natürlicher Lage im Körper), z. B. Stent-Implantation,

- ex vivo (außerhalb des Körpers, z. B. nach Entnahme), z. B. Spektroskopie am Tumor-Schnellschnitt,

- in vitro (im Reagenzglas), z. B. Züchtung von Stammzellen,

- in silico (am Computer): z. B. Computersimulation der Erregungsbildung und -leitung am Herzen für die Ableitung eines Therapievorschlages,

- nach der Dauer der Anwendung:

- Vorübergehend (weniger als $60 \mathrm{~min}$ ), z. B.: Operationsinstrumentarium,

- Kurzzeitig (bis zu 30 Tagen), z.B. Beatmung für die Dauer einer Woche,

- Langzeitig (mehr als 30 Tage), z. B. Herzschrittmacher,

- Ununterbrochen, z. B. künstliche Herzklappe (günstigenfalls lebenslang) [1].
Darüber hinaus findet man abhängig von der medizinischen Zielstellung verschiedene Einteilungen in Untergruppen. Beispielsweise ist eine Einteilung medizintechnischer Geräte nach Anwendung ionisierender (z. B. Computertomografie) oder nicht ionisierender Strahlung (z. B. Magnetresonanztomografie) möglich oder nach der Anzahl der vom Hersteller vorgesehenen Anwendungen (Mehrwegprodukt oder Einwegprodukt). Ein Teil der genannten Charakteristika hat Auswirkungen auf das potenzielle Risiko der Anwendung von Medizinprodukten und muss im Zulassungsprozess berücksichtigt werden (vgl. I4).

Eine übliche, jedoch nicht widerspruchs- und überschneidungsfreie Klassierung von Medizinprodukten verdeutlicht nachfolgend das Spektrum:

- Implantate (z. B. Herzschrittmacher, Herzklappen, Knochennägel, Ureterstents, Endoprothesen, Intraokularlinsen),

- Medizintechnische Geräte (z. B. Blutdruckmeßgeräte, Beatmungsgeräte, Infusionspumpen, Dialysegeräte, Ultraschallgeräte, Herz-Lungen-Maschinen, Sterilisatoren),

- Instrumente und Medicalprodukte (z. B. chirurgische Instrumente, chirurgisches Nahtmaterial, Bandagen, Inkontinenzhilfen, Kanülen),

- Hilfsmittel (z.B. Rollstühle, Exoprothesen, Gehhilfen, Kompressionsstrümpfe, Anti-Dekubitus-Systeme, siehe auch Hilfsmittelverzeichnis),

- Dentalprodukte (z. B. Inlays, Knochenersatzstoffe, künstliche Zähne),

- Produkte zur Empfängnisregelung (z. B. Kondome),

- in-vitro-Diagnostika und Diagnostikgeräte (z. B. Blutgasanalysegeräte, Blutkörperchenzählgeräte).

Der bei der Abgrenzung der Medizintechnik gegenüber anderen Technikbereichen im Vordergrund stehende Anwendungsbezug für die Heilkunde hat zur Folge, dass jedes technische System ein Medizinprodukt ist, wenn es die o.g. Definition des Medizinproduktegesetzes erfüllt. 